\title{
Suzuki type unique common fixed point theorem in partial metric spaces using $(\mathbf{C})$-condition
}

\author{
V. M. L. Hima Bindu' ${ }^{1}$ - G. N. V. Kishore ${ }^{1}$ K. P. R. Rao $^{2} \cdot$ Y. Phani $^{3}$
}

Received: 21 July 2016/ Accepted: 30 October 2016/Published online: 24 November 2016

(C) The Author(s) 2016. This article is published with open access at Springerlink.com

\begin{abstract}
In this paper, we obtain a Suzuki type unique common fixed point theorem using $C$-condition in partial metric spaces. In addition, we give an example which supports our main theorem.
\end{abstract}

Keywords Partial metric - Weakly compatible maps · Suzuki-type contraction $\cdot C$-condition

\section{Mathematics Subject Classification 54H25 - 47H10 . 54E50}

\section{Introduction}

The notion of a partial metric space was introduced by Matthews [12] as a part of the study of denotational semantics of data flow networks. In fact, it is widely

V. M. L. Hima Bindu

v.m.l.himabindu@gmail.com

G. N. V. Kishore

gnvkishore@kluniversity.in; kishore.apr2@gmail.com

K. P. R. Rao

kprrao2004@yahoo.com

Y. Phani

phaniyedlapalli23@gmail.com

1 Department of Mathematics, K L University, Vaddeswaram, Guntur 522 502, Andhra Pradesh, India

2 Department of Mathematics, Acharya Nagarjuna University, Nagarjuna Nagar, Guntur 522 510, Andhra Pradesh, India

3 Department of Basic Sciences, Shri Vishnu Engineering College for Women, Bhimavaram,

West Godavari 534 202, Andhra Pradesh, India recognized that partial metric spaces play an important role in constructing models in the theory of computation and domain theory in computer science (see [6]).

Matthews [12] and Romaguera [16] and Altun et al. [2] proved some fixed point theorems in partial metric spaces for a single map. For more works on fixed, common fixed point theorems in partial metric spaces, we refer [1, 3-5, 7-11, 13-15, 17-19]).

The aim of this paper is to prove a Suzuki type unique common fixed point theorem for four maps using (C)condition in partial metric spaces.

First, we give the following theorem of Suzuki [18].

Theorem 1.1 (See [18]) Let $(X, d)$ be a complete metric space and let $T$ be a mapping on $X$. Define a non-increasing function $\theta:[0,1) \rightarrow\left(\frac{1}{2}, 1\right]$ by

$\theta(r)= \begin{cases}1 & \text { if } 0 \leq r \leq \frac{(\sqrt{5}-1)}{2}, \\ (1-r) r^{-2} & \text { if } \frac{(\sqrt{5}-1)}{2} \leq r \leq 2^{-\frac{1}{2}}, \\ (1+r)^{-1} & \text { if } 2^{-\frac{1}{2}} \leq r<1 .\end{cases}$

Assume that there exists $r \in[0,1)$, such that

$\theta(r) d(x, T x) \leq d(x, y) \Rightarrow d(T x, T y) \leq r d(x, y)$

for all $x, y \in X$. Then, there exists a unique fixed point $z$ of T. Moreover, $\lim _{n} T^{n} x=z$ for all $x \in X$.

Definition 1.2 (See [11]) A mapping $T$ on a metric space $(X, d)$ is called a non-expensive mapping if

$d(T x, T y) \leq d(x, y), \quad \forall x, y \in X$.

Definition 1.3 (See [11]) A mapping $T$ on a metric space $(X, d)$ satisfies the $\mathrm{C}$-condition if 
$\frac{1}{2} d(x, T x) \leq d(x, y) \Rightarrow d(T x, T y) \leq d(x, y), \quad \forall x, y \in X$.

First, we recall some basic definitions and lemmas which play crucial role in the theory of partial metric spaces.

Definition 1.4 (See [12]) A partial metric on a nonempty set $X$ is a function $p: X \times X \rightarrow R^{+}$, such that for all $x, y, z \in X$ :

$$
\begin{aligned}
& \left(p_{1}\right) x=y \Leftrightarrow p(x, x)=p(x, y)=p(y, y), \\
& \left(p_{2}\right) p(x, x) \leq p(x, y), p(y, y) \leq p(x, y), \\
& \left(p_{3}\right) p(x, y)=p(y, x), \\
& \left(p_{4}\right) p(x, y) \leq p(x, z)+p(z, y)-p(z, z) .
\end{aligned}
$$

The pair $(X, p)$ is called a partial metric space (PMS).

If $p$ is a partial metric on $X$, then the function $p^{s}$ : $X \times X \rightarrow \mathbb{R}^{+}$given by

$p^{s}(x, y)=2 p(x, y)-p(x, x)-p(y, y)$,

is a metric on $X$.

Example 1.5 (See $[1,9,12])$ Consider $X=[0, \infty)$ with $p(x, y)=\max \{x, y\}$. Then, $(X, p)$ is a partial metric space. It is clear that $p$ is not a (usual) metric. Note that in this case, $p^{s}(x, y)=|x-y|$.

Example 1.6 (See [7]) Let $X=\{[a, b]: a, b, \in \mathbb{R}, a \leq b\}$ and define $p([a, b],[c, d])=\max \{b, d\}-\min \{a, c\}$. Then, $(X, p)$ is a partial metric space.

We now state some basic topological notions (such as convergence, completeness, and continuity) on partial metric spaces (see $[1,2,9,10,12]$.)

\section{Definition 1.7}

(i) A sequence $\left\{x_{n}\right\}$ in the PMS $(X, p)$ converges to the limit $x$ if and only if $p(x, x)=\lim _{n \rightarrow \infty} p\left(x, x_{n}\right)$.

(ii) A sequence $\left\{x_{n}\right\}$ in the PMS $(X, p)$ is called a Cauchy sequence if $\lim _{n, m \rightarrow \infty} p\left(x_{n}, x_{m}\right)$ exists and is finite.

(iii) A PMS $(X, p)$ is called complete if every Cauchy sequence $\left\{x_{n}\right\}$ in $X$ converges with respect to $\tau_{p}$, to a point $x \in X, \quad$ such that $p(x, x)=\lim _{n, m \rightarrow \infty} p\left(x_{n}, x_{m}\right)$.

The following lemma is one of the basic results in PMS $([1,2,9,10,12])$.

\section{Lemma 1.8}

(i) A sequence $\left\{x_{n}\right\}$ is a Cauchy sequence in the PMS $(X, p)$ if and only if it is a Cauchy sequence in the metric space $\left(X, p^{s}\right)$. (ii) A PMS $(X, p)$ is complete if and only if the metric space $\left(X, p^{s}\right)$ is complete. Moreover

$$
\begin{aligned}
& \lim _{n \rightarrow \infty} p^{s}\left(x, x_{n}\right)=0 \Leftrightarrow p(x, x)=\lim _{n \rightarrow \infty} p\left(x, x_{n}\right) \\
& =\lim _{n, m \rightarrow \infty} p\left(x_{n}, x_{m}\right) .
\end{aligned}
$$

Next, we give two simple lemmas which will be used in the proof of our main result. For the proofs, we refer to [1].

Lemma 1.9 Assume $x_{n} \rightarrow z$ as $n \rightarrow \infty$ in a PMS $(X, p)$, such that $p(z, z)=0$. Then, $\lim _{n \rightarrow \infty} p\left(x_{n}, y\right)=p(z, y)$ for every $y \in X$.

Lemma 1.10 Let $(X, p)$ be a PMS. Then

(A) If $p(x, y)=0$, then $x=y$.

(B) If $x \neq y$, then $p(x, y)>0$.

Remark 1.11 If $x=y, p(x, y)$ may not be 0 .

Definition 1.12 A pair ( $\mathrm{T}, \mathrm{g})$ is called weakly compatible pair if they commute at coincidence points.

Now, we prove our main result.

\section{Main result}

Theorem 2.1 Let $(X, p)$ be a partial metric space and let $S, T, f, g: X \rightarrow X$ be mappings satisfying

(2.1.1) $\frac{1}{2} \min \{p(f x, S x), p(g y, T y)\} \leq p(f x, g y) \quad$ implies that $\psi(p(S x, T y)) \leq \alpha(M(x, y))-\beta(M(x, y))$, for all $x, y$ in $X$, where $\psi, \alpha, \beta:[0, \infty) \rightarrow[0, \infty)$ are such that $\psi$ is an altering distance function, $\alpha$ is continuous, and $\beta$ is lower semi continuous, $\alpha(0)=\beta(0)=0$ and $\psi(t)-$ $\alpha(t)+\beta(t)>0$, for all $t>0$ and

$M(x, y)=\max \left\{\begin{array}{c}p(f x, g y), p(f x, S x), p(g y, T y), \\ \frac{1}{2}[p(f x, T y)+p(g y, S x)]\end{array}\right\}$,

(2.1.2) $S(X) \subseteq g(X), T(X) \subseteq f(X)$,

(2.1.3) either $f(X)$ or $g(X)$ is a complete subspace of $X$,

(2.1.4) the pairs $(f, S)$ and $(g, T)$ are weakly compatible.

Then, $S, T, f$ and $g$ have a unique common fixed point in $X$.

Proof Let $x_{0} \in X$ be arbitrary point in $X$. From (2.1.2), there exist sequences of $\left\{x_{n}\right\}$ and $\left\{y_{n}\right\}$ in $X$, such that $S x_{2 n}=g x_{2 n+1}=y_{2 n}$,

$T x_{2 n+1}=f x_{2 n+2}=y_{2 n+1}, \quad n=0,1,2, \ldots$.

Case (i): Assume that $y_{n} \neq y_{n+1}$ for all $n$.

Denote $p_{n}=p\left(y_{n}, y_{n+1}\right)$.

We show that $p_{n} \leq p_{n-1}, \quad n=1,2,3, \ldots$

Now 


$$
\begin{aligned}
\frac{1}{2} \min \left\{p\left(f x_{2 n}, S x_{2 n}\right), p\left(g x_{2 n+1}, T x_{2 n+1}\right)\right\} & \leq p\left(f x_{2 n}, S x_{2 n}\right) \\
& =p\left(f x_{2 n}, g x_{2 n+1}\right) .
\end{aligned}
$$

From (2.1.1), we get

$$
\begin{gathered}
\psi\left(p\left(S x_{2 n}, T x_{2 n+1}\right)\right) \leq \alpha\left(M\left(x_{2 n}, x_{2 n+1}\right)\right)-\beta\left(M\left(x_{2 n}, x_{2 n+1}\right)\right) . \\
\begin{array}{c}
M\left(x_{2 n}, x_{2 n+1}\right)=\max \left\{\begin{array}{c}
p\left(y_{2 n-1}, y_{2 n}\right), p\left(y_{2 n-1}, y_{2 n}\right), p\left(y_{2 n}, y_{2 n+1}\right), \\
\frac{1}{2}\left[p\left(y_{2 n-1}, y_{2 n+1}\right)+p\left(y_{2 n}, y_{2 n}\right)\right]
\end{array}\right\} \\
=\max \left\{p_{2 n-1}, p_{2 n}\right\}, \text { from }\left(p_{4}\right) .
\end{array}
\end{gathered}
$$

Hence,

$\psi\left(p_{2 n}\right) \leq \alpha\left(\max \left\{p_{2 n-1}, p_{2 n}\right\}\right)-\beta\left(\max \left\{p_{2 n-1}, p_{2 n}\right\}\right)$.

If $p_{2 n}$ is maximum, then we have $\psi\left(p_{2 n}\right) \leq \alpha\left(p_{2 n}\right)-$ $\beta\left(p_{2 n}\right)$, thus $\psi\left(p_{2 n}\right)-\alpha\left(p_{2 n}\right)+\beta\left(p_{2 n}\right) \leq 0$, which is a contradiction.

Hence $p_{2 n-1}$ is maximum. Thus

$\psi\left(p_{2 n}\right) \leq \alpha\left(p_{2 n-1}\right)-\beta\left(p_{2 n-1}\right)$

$<\psi\left(p_{2 n-1}\right)$.

Since $\psi$ is increasing, we have $p_{2 n} \leq p_{2 n-1}$.

Similarly, we can show that $p_{2 n-1} \leq p_{2 n-2}$.

Thus, $p_{n} \leq p_{n-1}, \quad n=1,2,3, \ldots$

Thus, $\left\{p_{n}\right\}$ is a non-increasing sequence of nonnegative real numbers and must converge to a real number, say, $l \geq 0$. Suppose $l>0$.

Letting $n \rightarrow \infty$ in (2), we get $\psi(l) \leq \alpha(l)-\beta(l)$.

Thus, $\psi(l)-\alpha(l)+\beta(l) \leq 0$, which is a contradiction. Hence, $l=0$.

Thus

$\lim _{n \rightarrow \infty} p\left(y_{n}, y_{n+1}\right)=0$.

Hence, from $\left(p_{2}\right)$, we get

$\lim _{n \rightarrow \infty} p\left(y_{n}, y_{n}\right)=0$.

By definition of $p^{s},(3)$, and (4), we get

$\lim _{n \rightarrow \infty} p^{s}\left(y_{n}, y_{n+1}\right)=0$.

Now, we prove that $\left\{y_{2 n}\right\}$ is a Cauchy sequence in $\left(X, p^{s}\right)$. On contrary, suppose that $\left\{y_{2 n}\right\}$ is not Cauchy.

There exist $\epsilon>0$ and monotone increasing sequences of natural numbers $\left\{2 m_{k}\right\}$ and $\left\{2 n_{k}\right\}$, such that $n_{k}>m_{k}$,

$p^{s}\left(y_{2 m_{k}}, y_{2 n_{k}}\right) \geq \epsilon$

and $p^{s}\left(y_{2 m_{k}}, y_{2 n_{k}-2}\right)<\epsilon$

From (6) and (7), we obtain

$$
\begin{aligned}
\epsilon & \leq p^{s}\left(y_{2 m_{k}}, y_{2 n_{k}}\right) \\
& \leq p^{s}\left(y_{2 m_{k}}, y_{2 n_{k}-2}\right)+p^{s}\left(y_{2 n_{k}-2}, y_{2 n_{k}-1}\right)+p^{s}\left(y_{2 n_{k}-1}, y_{2 n_{k}}\right) \\
& \leq \epsilon+p^{s}\left(y_{2 n_{k}-2}, y_{2 n_{k}-1}\right)+p^{s}\left(y_{2 n_{k}-1}, y_{2 n_{k}}\right) .
\end{aligned}
$$

Letting $k \rightarrow \infty$ and then using (5), we get

$\lim _{k \rightarrow \infty} p^{s}\left(y_{2 m_{k}}, y_{2 n_{k}}\right)=\epsilon$.

Hence, from definition of $p^{s}$ and (4), we have

$\lim _{k \rightarrow \infty} p\left(y_{2 m_{k}}, y_{2 n_{k}}\right)=\frac{\epsilon}{2}$.

Letting $k \rightarrow \infty$ and then using (8) and (5) in $\mid p^{s}\left(y_{2 n_{k}+1}\right.$, $\left.y_{2 m_{k}}\right)-p^{s}\left(y_{2 n_{k}}, y_{2 m_{k}}\right) \mid \leq p^{s}\left(y_{2 n_{k}+1}, y_{2 n_{k}}\right)$ we obtain

$\lim _{k \rightarrow \infty} p^{s}\left(y_{2 n_{k}+1}, y_{2 m_{k}}\right)=\epsilon$.

Hence, we have

$\lim _{k \rightarrow \infty} p\left(y_{2 n_{k}+1}, y_{2 m_{k}}\right)=\frac{\epsilon}{2}$.

Letting $k \rightarrow \infty$ and then using (8) and (5) in $\mid p^{s}\left(y_{2 n_{k}}\right.$, $\left.y_{2 m_{k}-1}\right)-p^{s}\left(y_{2 n_{k}}, y_{2 m_{k}}\right) \mid \leq p^{s}\left(y_{2 m_{k}-1}, y_{2 m_{k}}\right)$, we get

$\lim _{k \rightarrow \infty} p^{s}\left(y_{2 n_{k}}, y_{2 m_{k}-1}\right)=\epsilon$.

Hence, we have

$\lim _{k \rightarrow \infty} p\left(y_{2 n_{k}}, y_{2 m_{k}-1}\right)=\frac{\epsilon}{2}$.

Letting $k \rightarrow \infty$ and then using (12) and (5) in $\mid p^{s}\left(y_{2 m_{k}-1}\right.$, $\left.y_{2 n_{k}+1}\right)-p^{s}\left(y_{2 m_{k}-1}, y_{2 n_{k}}\right) \mid \leq p^{s}\left(y_{2 n_{k}+1}, y_{2 n_{k}}\right)$ we obtain

$\lim _{k \rightarrow \infty} p^{s}\left(y_{2 m_{k}-1}, y_{2 n_{k}+1}\right)=\epsilon$.

Hence, we get

$$
\lim _{k \rightarrow \infty} p\left(y_{2 m_{k}-1}, y_{2 n_{k}+1}\right)=\frac{\epsilon}{2} .
$$

If

$$
\frac{1}{2} \min \left\{p\left(y_{2 m_{k}-1}, y_{2 m_{k}}\right), p\left(y_{2 n_{k}}, y_{2 n_{k}+1}\right)\right\}>p\left(y_{2 m_{k}-1}, y_{2 n_{k}}\right),
$$

then letting $k \rightarrow \infty$, we get $0 \geq \frac{\epsilon}{2}$ from (3) and (13).

It is a contradiction. Hence $\frac{1}{2} \min \left\{p\left(y_{2 m_{k}-1}, y_{2 m_{k}}\right)\right.$, $\left.p\left(y_{2 n_{k}}, y_{2 n_{k}+1}\right)\right\} \leq p\left(y_{2 m_{k}-1}, y_{2 n_{k}}\right)=p\left(f x_{2 m_{k}}, g x_{2 n_{k}+1}\right)$.

From (2.1.1), we have 


$$
\begin{aligned}
& \psi\left(p\left(y_{2 m_{k}}, y_{2 n_{k}+1}\right)\right) \\
& =\psi\left(p\left(S x_{2 m_{k}}, T x_{2 n_{k}+1}\right)\right) \\
& \leq \alpha\left(\max \left\{\begin{array}{c}
p\left(y_{2 m_{k}-1}, y_{2 n_{k}}\right), p\left(y_{2 m_{k}-1}, y_{2 m_{k}}\right), p\left(y_{2 n_{k}}, y_{2 n_{k}+1}\right), \\
\frac{1}{2}\left[p\left(y_{2 m_{k}-1}, y_{2 n_{k}+1}\right)+p\left(y_{2 n_{k}}, y_{2 m_{k}}\right)\right]
\end{array}\right\}\right) \\
& -\beta\left(\max \left\{\begin{array}{c}
p\left(y_{2 m_{k}-1}, y_{2 n_{k}}\right), p\left(y_{2 m_{k}-1}, y_{2 m_{k}}\right), p\left(y_{2 n_{k}}, y_{2 n_{k}+1}\right), \\
\frac{1}{2}\left[p\left(y_{2 m_{k}-1}, y_{2 n_{k}+1}\right)+p\left(y_{2 n_{k}}, y_{2 m_{k}}\right)\right]
\end{array}\right\}\right) .
\end{aligned}
$$

Letting $k \rightarrow \infty$ and then using (11), (13), (3), (15), and (9), we have

$$
\begin{aligned}
\psi\left(\frac{\epsilon}{2}\right) \leq & \alpha\left(\max \left\{\frac{\epsilon}{2}, 0,0, \frac{1}{2}\left[\frac{\epsilon}{2}+\frac{\epsilon}{2}\right]\right\}\right) \\
& -\beta\left(\max \left\{\frac{\epsilon}{2}, 0,0, \frac{1}{2}\left[\frac{\epsilon}{2}+\frac{\epsilon}{2}\right]\right\}\right) \\
= & \alpha\left(\frac{\epsilon}{2}\right)-\beta\left(\frac{\epsilon}{2}\right) \\
< & \psi\left(\frac{\epsilon}{2}\right),
\end{aligned}
$$

which is a contradiction. Hence, $\left\{y_{2 n}\right\}$ is Cauchy.

In addition, $\left|p^{s}\left(y_{2 n+1}, y_{2 m+1}\right)-p^{s}\left(y_{2 n}, y_{2 m}\right)\right| \leq p^{s}\left(y_{2 n+1}\right.$, $\left.y_{2 n}\right)+p^{s}\left(y_{2 m}, y_{2 m+1}\right)$.

Letting $n, m \rightarrow \infty$, we have

$\lim _{n, m \rightarrow \infty} p^{s}\left(y_{2 n+1}, y_{2 m+1}\right)=0$.

Hence, $\left\{y_{2 n+1}\right\}$ is Cauchy. Thus $\left\{y_{n}\right\}$ is a Cauchy sequence in $\left(X, p^{s}\right)$.

Hence, we have $\lim _{n, m \rightarrow \infty} p^{s}\left(y_{n}, y_{m}\right)=0$.

Now, from the definition of $p^{s}$ and from (4), we obtain

$$
\lim _{n, m \rightarrow \infty} p\left(y_{n}, y_{m}\right)=0 \text {. }
$$

Therefore, $\left\{y_{n}\right\}$ is Cauchy sequence in $X$.

Suppose $g(X)$ is complete.

Since $y_{2 n}=S x_{2 n}=g x_{2 n+1}$, it follows $\left\{y_{2 n}\right\} \subseteq g(X)$ is a Cauchy sequence in the complete metric space $\left(g(X), p^{s}\right)$, it follows that $\left\{y_{2 n}\right\}$ converges in $\left(g(X), p^{s}\right)$.

Thus, $\lim _{n \rightarrow \infty} p^{s}\left(y_{2 n}, u\right)=0$ for some $u \in g(X)$.

That is, $y_{2 n} \rightarrow u=g t \in g(X)$ for some $t \in X$.

Since $\left\{y_{n}\right\}$ is Cauchy in $X$ and $\left\{y_{2 n}\right\} \rightarrow u$, it follows that $\left\{y_{2 n+1}\right\} \rightarrow u$.

From Lemma (1.2.5), we get

$$
p(u, u)=\lim _{n \rightarrow \infty} p\left(y_{2 n+1}, u\right)=\lim _{n \rightarrow \infty} p\left(y_{2 n}, u\right)=\lim _{n, m \rightarrow \infty} p\left(y_{n}, y_{m}\right) \text {. }
$$

From (16) and (17), we obtain

$$
p(u, u)=\lim _{n \rightarrow \infty} p\left(y_{2 n+1}, u\right)=\lim _{n \rightarrow \infty} p\left(y_{2 n}, u\right)=0 .
$$

Now, we claim that, for each $n \geq 1$, at least, one of the following assertions holds: $\frac{1}{2} p\left(y_{2 n-1}, y_{2 n}\right) \leq p\left(y_{2 n-1}, u\right)$ or $\frac{1}{2} p\left(y_{2 n}, y_{2 n+1}\right) \leq p\left(y_{2 n}, u\right)$.

On the contrary, suppose that $\frac{1}{2} p\left(y_{2 n-1}, y_{2 n}\right)>p\left(y_{2 n-1}, u\right)$ and $\frac{1}{2} p\left(y_{2 n}, y_{2 n+1}\right)>p\left(y_{2 n}, u\right)$

for some $n \geq 1$.

Then we have

$$
\begin{aligned}
p_{2 n-1}=p\left(y_{2 n-1}, y_{2 n}\right) & \leq p\left(y_{2 n-1}, u\right)+p\left(u, y_{2 n}\right)-p(u, u) \\
& <\frac{1}{2}\left[p\left(y_{2 n-1}, y_{2 n}\right)+p\left(y_{2 n}, y_{2 n+1}\right)\right] \\
& \leq \frac{1}{2}\left[p_{2 n-1}+p_{2 n}\right] \\
& \leq p_{2 n-1},
\end{aligned}
$$

which is a contradiction, and so, the claim holds.

Sub case $(a)$ : Suppose $\frac{1}{2} p\left(y_{2 n-1}, y_{2 n}\right) \leq p\left(y_{2 n-1}, u\right)$.

Suppose $T t \neq u$.

We have

$$
\begin{aligned}
\frac{1}{2} \min \left\{p\left(f x_{2 n}, S x_{2 n}\right), p(g t, T t)\right\} & \leq \frac{1}{2} p\left(f x_{2 n}, S x_{2 n}\right) \\
& =\frac{1}{2} p\left(y_{2 n-1}, y_{2 n}\right) \\
& \leq p\left(y_{2 n-1}, u\right) \\
& =p\left(f x_{2 n}, g t\right) .
\end{aligned}
$$

From (2.1.1), we get

$$
\begin{aligned}
\psi\left(p\left(S x_{2 n}, T t\right)\right) \leq & \alpha\left(M\left(x_{2 n}, t\right)\right)-\beta\left(M\left(x_{2 n}, t\right)\right) \\
\leq & \alpha\left(\max \left\{\begin{array}{c}
p\left(f x_{2 n}, g t\right), p\left(f x_{2 n}, S x_{2 n}\right), p(g t, T t), \\
\frac{1}{2}\left[p\left(f x_{2 n}, T t\right)+p\left(g t, S x_{2 n}\right)\right]
\end{array}\right\}\right) \\
& -\beta\left(\max \left\{\begin{array}{c}
p\left(f x_{2 n}, g t\right), p\left(f x_{2 n}, S x_{2 n}\right), p(g t, T t), \\
\frac{1}{2}\left[p\left(f x_{2 n}, T t\right)+p\left(g t, S x_{2 n}\right)\right]
\end{array}\right\}\right) .
\end{aligned}
$$

Letting $n \rightarrow \infty$ and using (17), (18), we get

$$
\begin{aligned}
\psi(p(u, T t)) \leq \alpha & \left(\max \left\{\begin{array}{c}
p(u, g t), p(u, u), p(g t, T t), \\
\frac{1}{2}[p(u, T t)+p(g t, u)]
\end{array}\right)\right. \\
& -\beta\left(\max \left\{\begin{array}{c}
p(u, g t), p(u, u), p(g t, T t), \\
\frac{1}{2}[p(u, T t)+p(g t, u)]
\end{array}\right\}\right) \\
= & \left(\max \left\{\begin{array}{c}
p(u, u), p(u, u), p(u, T t), \\
\frac{1}{2}[p(u, T t)+p(u, u)]
\end{array}\right)\right. \\
& -\beta\left(\max \left\{\begin{array}{c}
p(u, u), p(u, u), p(u, T t), \\
\frac{1}{2}[p(u, T t)+p(u, u)]
\end{array}\right\}\right) \\
\leq & \alpha(p(u, T t))-\beta(p(u, T t))<\psi(p(u, T t)) .
\end{aligned}
$$


It is a contradiction. Hence, $T t=u=g t$.

Since the pair $(g, T)$ is weakly compatible, we have $g u=T u$.

Suppose $T u \neq u$.

Since $\frac{1}{2} \min \left\{p\left(f x_{2 n}, S x_{2 n}\right), p(g u, T u)\right\} \leq p\left(f x_{2 n}, g u\right)$, from (2.1.1), we get

$$
\begin{aligned}
\psi\left(p\left(S x_{2 n}, T u\right)\right) \leq & \alpha\left(\max \left\{\begin{array}{c}
p\left(f x_{2 n}, g u\right), p\left(f x_{2 n}, S x_{2 n}\right), p(g u, T u), \\
\frac{1}{2}\left[p\left(f x_{2 n}, T u\right)+p\left(g u, S x_{2 n}\right)\right]
\end{array}\right\}\right) \\
& -\beta\left(\max \left\{\begin{array}{c}
p\left(f x_{2 n}, g u\right), p\left(f x_{2 n}, S x_{2 n}\right), p(g u, T u), \\
\frac{1}{2}\left[p\left(f x_{2 n}, T u\right)+p\left(g u, S x_{2 n}\right)\right]
\end{array}\right\}\right) .
\end{aligned}
$$

Letting $n \rightarrow \infty$, we have

$$
\begin{aligned}
\psi(p(u, T u)) \leq & \alpha\left(\max \left\{\begin{array}{c}
p(u, g u), p(u, u), p(g u, T u), \\
\frac{1}{2}[p(u, T u)+p(g u, u)]
\end{array}\right\}\right) \\
& -\beta\left(\max \left\{\begin{array}{c}
p(u, g u), p(u, u), p(g u, T u), \\
\frac{1}{2}[p(u, T u)+p(g u, u)]
\end{array}\right\}\right) \\
\leq & \alpha(p(u, T u))-\beta(p(u, T u)) \\
< & \psi(p(u, T u)),
\end{aligned}
$$

which is a contradiction.

Hence, $T u=u$.

Therefore, $u=T u=g u$.

Since $T(X) \subseteq f(X)$, then there exists $v \in X$, such that $T u=f v=u$.

Suppose $S v \neq f v$.

Since $\quad \frac{1}{2} \min \{p(f v, S v), p(g u, T u)\} \leq p(f v, g u), \quad$ from (2.1.1), we have

$$
\begin{aligned}
\psi(p(S v, f v)) & =\psi(p(S v, T u)) \\
& \leq \alpha(M(v, u))-\beta(M(v, u)) \\
& \leq \alpha\left(\max \left\{\begin{array}{c}
p(f v, g u), p(f v, S v), p(g u, T u), \\
\frac{1}{2}[p(f v, T u)+p(g u, S v)]
\end{array}\right\}\right) \\
& -\beta\left(\max \left\{\begin{array}{c}
p(f v, g u), p(f v, S v), p(g u, T u), \\
\frac{1}{2}[p(f v, T u)+p(g u, S v)]
\end{array}\right\}\right) \\
& \leq \alpha(p(S v, T u))-\beta(p(S v, T u)) \\
& <\psi(p(S v, T u)) \\
& =\psi(p(S v, f v)) .
\end{aligned}
$$

Hence, $S v=f v=u$.
Since the pair $(f, S)$ is weakly compatible, we have $f u=S u$.

Suppose $S u \neq u$.

Since $\quad \frac{1}{2} \min \{p(f u, S u), p(g t, T t)\} \leq p(f u, g t), \quad$ from (2.1.1), we have

$$
\begin{aligned}
\psi(p(S u, u))= & \psi(p(S u, T t)) \\
\leq & \alpha\left(\max \left\{\begin{array}{c}
p(f u, g t), p(f u, S u), p(g t, T t), \\
\frac{1}{2}[p(f u, T t)+p(g t, S u)]
\end{array}\right\}\right) \\
& -\beta\left(\max \left\{\begin{array}{c}
p(f u, g t), p(f u, S u), p(g t, T t), \\
\frac{1}{2}[p(f u, T t)+p(g t, S u)]
\end{array}\right\}\right) \\
\leq & \alpha(p(S u, T t))-\beta(p(S u, T t))<\psi(p(S u, u)) .
\end{aligned}
$$

Is a contradiction. Hence, $u=S u=f u$.

Thus, $T u=g u=S u=f u=u$.

Hence, $u$ is a common fixed point of $S, T, f$ and $g$.

Let $w$ be another common fixed point of $S, T, f$ and $g$.

Since $\frac{1}{2} \min \{p(f u, S u), p(g w, T w)\} \leq p(f u, g w), \quad$ from (2.1.1), we obtain

$$
\begin{aligned}
\psi(p(u, w))= & \psi(p(S u, T w)) \\
\leq & \alpha\left(\max \left\{\begin{array}{c}
p(f u, g w), p(f u, S u), p(g w, T w), \\
\frac{1}{2}[p(f u, T w)+p(g w, S u)]
\end{array}\right\}\right) \\
& -\beta\left(\max \left\{\begin{array}{c}
p(f u, g w), p(f u, S u), p(g w, T w), \\
\frac{1}{2}[p(f u, T w)+p(g w, S u)]
\end{array}\right\}\right) \\
\leq & \alpha\left(\max \left\{\begin{array}{c}
p(u, w), p(u, u), p(w, w), \\
\frac{1}{2}[p(u, w)+p(w, u)]
\end{array}\right\}\right) \\
& -\beta\left(\max \left\{\begin{array}{c}
p(u, w), p(u, u), p(w, w), \\
\frac{1}{2}[p(u, w)+p(w, u)]
\end{array}\right\}\right) \\
\leq & \alpha(p(u, w))-\beta(p(u, w)) \\
< & \psi(p(u, w)),
\end{aligned}
$$

which is a contradiction. Hence, $u=w$.

Thus, $u$ is the unique common fixed point of $S, T, f$ and g.

Sub case (b) : Suppose $\frac{1}{2} p\left(y_{2 n}, y_{2 n+1}\right) \leq p\left(y_{2 n}, u\right)$.

In this case, also, we can prove that $u$ is the unique common fixed point of $S, T, f$ and $g$ by proceeding as in Subcase(a).

Case(ii): Suppose $y_{2 m}=y_{2 m+1}$ for some $\mathrm{m}$.

Assume that $y_{2 m+1} \neq y_{2 m+2}$. 
$M\left(x_{2 m+2}, x_{2 m+1}\right)=\max \left\{\begin{array}{c}p\left(y_{2 m+1}, y_{2 m}\right), p\left(y_{2 m+1}, y_{2 m+2}\right), p\left(y_{2 m}, y_{2 m+1}\right), \\ \frac{1}{2}\left[p\left(y_{2 m+1}, y_{2 m+1}\right)+p\left(y_{2 m}, y_{2 m+2}\right)\right]\end{array}\right\}$.

However, $\quad p\left(y_{2 m+1}, y_{2 m}\right)=p\left(y_{2 m+1}, y_{2 m+1}\right) \leq p\left(y_{2 m+1}\right.$, $\left.y_{2 m+2}\right)$, from $\left(p_{2}\right)$ and

$$
\begin{aligned}
& \frac{1}{2}\left[p\left(y_{2 m+1}, y_{2 m+1}\right)+p\left(y_{2 m}, y_{2 m+2}\right)\right] \\
& \leq \leq \frac{1}{2}\left[p\left(y_{2 m}, y_{2 m+1}\right)+p\left(y_{2 m+1}, y_{2 m+2}\right)\right], \text { from }\left(p_{4}\right) \\
& \quad \leq \frac{1}{2}\left[p\left(y_{2 m+1}, y_{2 m+2}\right)+p\left(y_{2 m+1}, y_{2 m+2}\right)\right] \\
& \quad=p\left(y_{2 m+1}, y_{2 m+2}\right) .
\end{aligned}
$$

Hence, $M\left(x_{2 m+2}, x_{2 m+1}\right)=p\left(y_{2 m+1}, y_{2 m+2}\right)$.

$$
\begin{aligned}
& \text { Since } \frac{1}{2} \min \left\{p\left(f x_{2 m+2}, S x_{2 m+2}\right), p\left(g x_{2 m+1}, T x_{2 m+1}\right)\right\} \\
& \quad \leq p\left(g x_{2 m+1}, T x_{2 m+1}\right) \\
& \quad=p\left(f x_{2 m+2}, g x_{2 m+1}\right),
\end{aligned}
$$

from (2.1.1), we get

$$
\begin{aligned}
\psi\left(p\left(y_{2 m+2}, y_{2 m+1}\right)\right) & =\psi\left(p\left(S x_{2 m+2}, T x_{2 m+1}\right)\right) \\
& \leq \alpha\left(M\left(x_{2 m+2}, x_{2 m+1}\right)\right)-\beta\left(M\left(x_{2 m+2}, x_{2 m+1}\right)\right) \\
& =\alpha\left(p\left(y_{2 m+2}, y_{2 m+1}\right)\right)-\beta\left(p\left(y_{2 m+2}, y_{2 m+1}\right)\right) \\
& <\psi\left(p\left(y_{2 m+2}, y_{2 m+1}\right)\right) .
\end{aligned}
$$

It is a contradiction. Hence, $y_{2 m+2}=y_{2 m+1}$.

Continuing in this way, we can conclude that $y_{n}=y_{n+k}$ for all $k>0$.

Thus, $\left\{y_{n}\right\}$ is a Cauchy sequence.

The rest of the proof follows as in Case(i).

The following example illustrates our Theorem 2.1

Example 2.2 Let $X=[0,1]$ and $p(x, y)=\max \{x, y\}$ for all $x, y \in X$. Let $f, g, S, T: X \rightarrow X, f(x)=\frac{x}{2}, g(x)=$ $\frac{x}{3}, S(x)=\frac{x}{4}$ and $T(x)=\frac{x}{6}$, Let $\psi, \alpha, \beta:[0, \infty) \rightarrow[0, \infty)$ be defined by $\psi(t)=4 t, \alpha(t)=7 t$ and $\beta(t)=\frac{7 t}{2}$. Clearly, $\psi$ is an altering distance function and $\alpha$ is continuous and $\beta$ is lower semi continuous, $\alpha(0)=\beta(0)=0$ and $\psi(t)-\alpha(t)+$ $\beta(t)=\frac{t}{2}>0$, for all $t>0$.

Now

$$
\begin{aligned}
\frac{1}{2} \min \{p(f x, S x), p(g y, T y)\} & =\frac{1}{2} \min \{\max \{f x, S x\}, \max \{g y, T y\}\} \\
& =\frac{1}{2} \min \left\{\max \left\{\frac{x}{2}, \frac{x}{4}\right\}, \max \left\{\frac{y}{3}, \frac{y}{6}\right\}\right\} \\
& =\frac{1}{2} \min \left\{\frac{x}{2}, \frac{y}{3}\right\} \\
& \leq \frac{1}{2} \max \left\{\frac{x}{2}, \frac{y}{3}\right\} \\
& \leq p(f x, g y) .
\end{aligned}
$$

$$
\begin{aligned}
\psi(p(S x, T y)) & =4 p(S x, T y) \\
& =4 \max \left\{\frac{x}{4}, \frac{y}{6}\right\} \\
& =4 \times \frac{1}{2} \max \left\{\frac{x}{2}, \frac{y}{3}\right\} \\
& =2 p(f x, g y) \\
& \leq 2 M(x, y) \\
& \leq 7 M(x, y)-\frac{7}{2} M(x, y) .
\end{aligned}
$$

So

$\psi(p(S x, T y)) \leq \alpha(M(x, y))-\beta(M(x, y))$

Therefore, all of the conditions of Theorem 2.1 are satisfied and 0 is the unique common fixed point of $S, T, f$ and $g$.

Acknowledgements The authors are thankful to referee for his valuable suggestions.

Open Access This article is distributed under the terms of the Creative Commons Attribution 4.0 International License (http://crea tivecommons.org/licenses/by/4.0/), which permits unrestricted use, distribution, and reproduction in any medium, provided you give appropriate credit to the original author(s) and the source, provide a link to the Creative Commons license, and indicate if changes were made.

\section{References}

1. Abdeljawad, T., Karapınar, E., Tas, K.: Existence and uniqueness of a common fixed point on partial metric spaces. Appl. Math. Lett. 24(11), 1900-1904 (2011)

2. Altun, I., Sola, F., Simsek, H.: Generalized contractions on partial metric spaces. Topol. Appl. 157(18), 2778-2785 (2010)

3. Altun, I., Erduran, A.: Fixed point theorems for monotone mappings on partial metric spaces. Fixed Point Theory and Applications Article ID 508730, p. 10 (2011). doi:10.1155/2011/508730

4. Aydi, H.: Fixed point results for weakly contractive mappings in ordered partial metric spaces. J. Adv. Math. Stud. 4(2), 01-12 (2011)

5. Fadail, Z.M., Ahmed, A.G.B., Ansar, A.H., Radenovic, S., Rajovic, M.: Some common fixed point results of mappings in 0 -s-complete metric-like spaces via new functions. Appl. Math. Sci. 9(83), 5009-5027 (2015)

6. Heckmann, R.: Approximation of metric spaces by partial metric spaces. Appl. Categ. Struct. 7(1-2), 71-83 (1999)

7. Ilić, D., Pavlović, V., Rakočević, V.: Some new extensions of Banach's contraction principle to partial metric spaces. Appl. Math. Lett. 24(8), 1326-1330 (2011)

8. Kadelburg, Z., Radenovic, S.: Fixed points under $\alpha-\beta$ conditions in ordered partial metric spaces. Int. J. Anal. Appl. 5(1), 91-101 (2014)

9. Karapınar, E., Erhan, I.M.: Fixed point theorems for operators on partial metric spaces. Appl. Math. Lett. 24(11), 1894-1899 (2011)

10. Karapinar, E.: Generalizations of Caristi Kirk's theorem on partial metric spaces. Fixed Point Theory Appl. 2011, 4 (2011)

11. Karapinar, E., Erhan, I.M., Aksoy, U.: Weak $\psi$-contractions on partially ordered metric spaces and applications to boundary 
value problems. Bound. Value Probl. 2014, 149 (2014). doi:10. 1186/s13661-014-0149-8

12. Matthews, S.G.: Partial metric topology. In: Proceedings of the 8th Summer Conference on General Topology and Applications 1994. vol. 728, pp. 183-197. Annals of the New York Academy of Sciences (1994)

13. Mustafa, Z., Huang, H., Radenovic, S.: Some remarks on the paper "Some fixed point generalizations are not real generalizations". J. Adv. Math. Stud. 05/11/2015 (to appear)

14. Radenovic, S.: Classical fixed point results in 0-complete partial metric spaces via cyclic-type extension. Allahabad Math. Soc. 31(1), 39-55 (2016)

15. Rao, K.P.R., Kishore, G.N.V.: A unique common fixed point theorem for four maps under $\psi-\phi$ contractive condition in partial metric spaces. Bull. Math. Anal. Appl. 3(3), 56-63 (2011)
16. Romaguera, S.: A Kirk type characterization of completeness for partial metric spaces. Fixed Point Theory Appl. Article ID 493298, p. 6 (2010)

17. Shukla, S., Radenovic, S., Vetro, C.: Set-valued Hardy-Rogers type ontraction in 0-complete partial metric spaces. Int. J. Math. Math. Sci. Article ID 652925, p. 9 (2014)

18. Suzuki, T.: A generalized Banach contraction principle which characterizes metric completeness. Proc. Am. Math. Soc. 136, 1861-1869 (2008)

19. Valero, O.: On Banach fixed point theorems for partial metric spaces. Appl. Gen. Topol. 6(2), 229-240 (2005) 